\title{
FREQUENCY WEIGHTED CONTROLLER ORDER REDUCTION (PART I)
}

\author{
Roozbeh Sadeghian — Paknosh Karimaghaee — Alireza Khayatian *
}

\begin{abstract}
In this paper, a new method for controller reduction of linear time invariant systems is presented. The method is based on newly defined controllability and observability grammians which are calculated from input to state and state to output characteristics of the controller in a certain frequency domain. These grammians are defined for the closed loop system to keep the performance of original controller. The main idea of this method is based on Moores model reduction. The relation of this method with weighted frequency model reduction of Enns will be described by a commutative diagram. The stability property of the new method is investigated. It is shown that the stability for two sided weights can be preserved under certain conditions. The simulation results show the effectiveness of this novel technique.
\end{abstract}

K e y w ords: frequency-domain, controller reduction, LTI systems, continuous-time

\section{INTRODUCTION}

There are many controller design methods such as $L Q R, H_{2}, H_{\infty}, \ldots$ for high order models which often lead to high order controllers. As the reduced order controllers are easier to understand and have less complication, it is desired to reduce the order of such high order controllers. Many controller reduction methods for linear systems have recently been proposed [1-7]. The main idea of many of these methods is that the reduced order controller has the same performance as the full order controller when the closed loop system is considered in a certain bandwidth. Based on [8], there are two main controller reduction methods, which are known as direct and indirect method. In direct method, a low-order controller is designed by minimizing a quadratic performance index subject to a certain order constraint. In indirect method, first a high-order controller is found then it is simplified. Most controller reduction methods and also the new technique in this paper are based on indirect approach.

One of the main model and controller reduction techniques is frequency weighted method which was first introduced by Enns [8] and further developed by others [1-5]. Enns showed that by selecting certain weights, the accuracy of model reduction could be improved. Enns weights should be one sided for stability property and he could not show that two sided weights can make the system stable. One important contribution of this paper is the stability result for two sided weights which further completes the Enns work. Others eg $[3,5]$ have also tried to develop new methods for two sided weights which can keep the stability of the reduced model. Yousefi and Lohmann [10] have considered the instability in frequency weighted controller reduction methods based on similarity transformations. Houlis and Sreeram [7] have tried to approximate a low order controller for problems in a doublesided frequency weighted form. Ghafoor and Sreeram [11] have proposed a complete survey of frequency weighted balanced model and controller reduction techniques.

The main idea of this paper is based on Moores model reduction approach [12] but in frequency domain. Moreover, unlike to the Moore method which is for an open loop plant here the closed loop system is considered. From a physical interpretation view, the input to state and the state to output energies of the transfer function to be reduced is considered and new controllability and observability grammians for the system in the closed loop framework are defined. Because these grammians are based on a physical interpretation, the results of controller reduction with these grammians are better than other controller reduction techniques. One interesting result is that by defining new weights, these grammians can be related to Enns frequency weighted grammians.

It is obvious that most of the systems are designed to work in a certain frequency domain. This method enables the designer to select the frequency range of interest for the best performance.

The structure of the paper is as follows; model reduction based on balanced realization and frequency weighted model reduction together with the recent reduction techniques is briefly reviewed in section 2. In section 3 , the new controllability and observability grammians for the controller with closed loop consideration is introduced. The relation of new grammians to the grammians of the overall closed loop system, the properties of the new method and its relation to Enns frequency weighted method, in addition to new stability results are

\footnotetext{
* School of Electrical and Computer Engineering, Shiraz University, Shiraz, Iran, (corresponding author: Paknoosh Karimaghee, kaghaee@shirazu.ac.ir)
} 


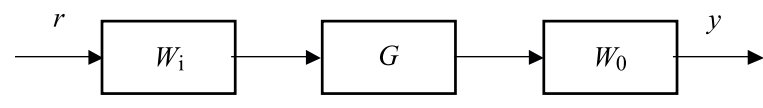

Fig. 1. A plant with two sided weights

discussed in section 4. Finally, simulation results are represented in section 5 and conclusion is given in section 6 .

\section{MODEL REDUCTION TECHNIQUES}

Consider an nth-order linear time-invariant asymptotically stable continuous system with minimal realization $(A, B, C)$ with system equations as

$$
\begin{aligned}
& \dot{x}(t)=A x(t)+B u(t), \\
& y(t)=C x(t)
\end{aligned}
$$

where $u \in R^{p}, y \in R^{q}, x \in R^{n}$ are the input, output, and state, respectively. Also, $A \in R^{n \times n}, B \in R^{n \times p}, C \in$ $R^{q \times n}$ are real valued matrices.

The controllability and observability grammians of system (2.1) can be defined respectively as

$$
\begin{aligned}
& P=\int_{0}^{\infty} e^{A t} B B^{\top} e^{A^{\top} t} \mathrm{~d} t, \\
& Q=\int_{0}^{\infty} e^{A^{\top} t} C^{\top} C e^{A t} \mathrm{~d} t .
\end{aligned}
$$

For an asymptotically stable system, the following Lyapunov equations are satisfied by the grammian matrices of $(2.2)$.

$$
\begin{aligned}
& A P+P A^{\top}=-B B^{\top}, \\
& A^{\top} Q+Q A=-C^{\top} C .
\end{aligned}
$$

It has been shown [12] that similarity transformation can be found such that the system is internally balanced, that is, the matrices $P, Q$ are equal and diagonal

$$
P=Q=\Sigma=\operatorname{diag}\left\{\sigma_{1}, \sigma_{2}, \ldots, \sigma_{n}\right\}
$$

where $\sigma_{i}>\sigma_{i+1}, i=1,2, \ldots, n-1$ are the grammians singular values and are invariant under similarity transformation. Based on the order of magnitude of singular values, this balanced system and its corresponding grammians can be partitioned as below

$$
\begin{aligned}
& A=\left[\begin{array}{ll}
A_{11} & A_{12} \\
A_{21} & A_{22}
\end{array}\right], \quad B=\left[\begin{array}{l}
B_{1} \\
B_{2}
\end{array}\right], \\
& C=\left[\begin{array}{ll}
C_{1} & C_{2}
\end{array}\right], \Sigma=\left[\begin{array}{cc}
\Sigma_{1} & 0 \\
0 & \Sigma_{2}
\end{array}\right] .
\end{aligned}
$$

It has been showed that if $\sigma_{i} \gg \sigma_{i+1}$, the subsystem $\left(A_{11}, B_{1}, C_{1}\right)$ is a good reduced order approximation of the main full order system $(A, B, C)$. This technique is called Balance Truncation (BT).

In Enns model reduction approach, instead of using equation (2.2) to define the controllability and observability grammians, input weight $W_{i}$ and output weight $W_{0}$ are added to the system and frequency weighted grammians are introduced. Figure 1 shows the structure of input and output weights on system. The goal is to minimize $\left\|W_{0}\left(G-G_{r}\right) W_{i}\right\|$ instead of $\left\|G-G_{r}\right\|$ for a specified norm $\|\cdot\|$, where $G$ and $G_{r}$ are the transfer functions of full and reduced order systems. This technique provides flexibility in selecting important frequency range.

Consider the input and output weights realization as $W_{i}=\left(A_{i}, B_{i}, C_{i}, D_{i}\right), W_{0}=\left(A_{0}, B_{0}, C_{0}, D_{0}\right)$; then, the realizations of $G W_{i}, W_{0} G$ is

$$
G W_{i}=\left[\begin{array}{l|l}
\bar{A}_{i} & \bar{B}_{i} \\
\hline \bar{C}_{i} & \bar{D}_{i}
\end{array}\right], \quad W_{0} G=\left[\begin{array}{l|l}
\bar{A}_{0} & \bar{B}_{0} \\
\hline \bar{C}_{0} & \bar{D}_{0}
\end{array}\right]
$$

The Lyapunov equation for the realizations of (2.6) will be

$$
\bar{A}_{i} \tilde{P}+\tilde{P} \bar{A}_{i}=-\bar{B}_{i} \bar{B}_{i}^{\top}, \quad \bar{A}_{0}^{\top} \tilde{Q}+\tilde{Q} \bar{A}_{0}=-\bar{C}_{0}^{\top} \bar{C}_{0} .
$$

The solutions of eqs. (2.7) are the frequency weighted controllability and observability grammians, and can be written as

$$
\tilde{P}=\left[\begin{array}{ll}
P_{E} & P_{12} \\
P_{12}^{\top} & P_{22}
\end{array}\right], \quad \tilde{P}=\left[\begin{array}{cc}
Q_{E} & Q_{12} \\
Q_{12}^{\top} & Q_{22}
\end{array}\right]
$$

For frequency weighted model reduction, $P_{E}$ and $Q_{E}$ as alternative controllability and observability grammians are equalized and diagonolized in a similar procedure as above to obtain a reduced order model. This method of balanced truncation is called frequency weighted balance truncation (FWBT). The method has been shown to preserve reduced order model stability for one sided weights $\left(W_{i}=I, W_{0} \neq I\right.$ or $\left.W_{i} \neq I, W_{0}=I\right)[9]$ but no results are claimed for two sided weights. Enns has also shown that for controller reduction the weights $W_{0}=(I+G K)^{-1} G, W_{i}=I$ or $W_{i}=G(I+G K)^{-1}$, $W_{0}=I$ are good choices for controller reduction where $K$ is the controller transfer function. These weights were obtained by using small gain theorem.

Lin and Chiu [1] showed that for FWBT, new grammians can be defined with two sided weights which can preserve stability. The grammians can be chosen as

$$
\begin{aligned}
P_{L} & =P_{E}-P_{12} P_{22}^{-1} P_{12}^{\top}, \\
Q_{L} & =Q_{E}-Q_{12}^{\top} Q_{22}^{-1} Q_{12}
\end{aligned}
$$

Where $P_{E}, P_{12}, P_{22}, Q_{E}, Q_{12}$ and $Q_{22}$ are defined in (2.8).

This method guarantees the stability of reduced order model and can be used for two sided weights. The disadvantage of Lin method is that in forming $G W_{i}$ and 


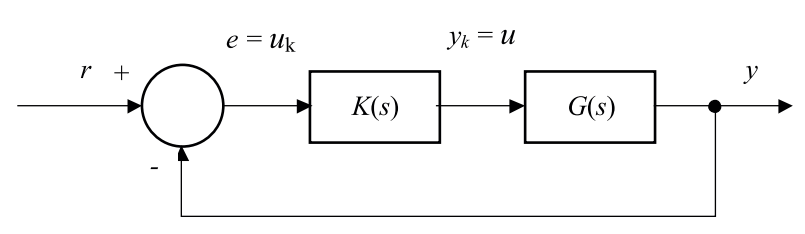

Fig. 2. The closed loop system

$W_{0} G$ no pole-zero cancellation should occur and unfortunately with Enns weights for controller reduction this always happens.

In [3], a new method was introduced to solve the problem of pole-zero cancellation in Lin's method. In this method, one can define new symmetric matrices as $X=-A P_{E}-P_{E} A^{\top}$ and $Y=-A^{\top} Q_{E}-Q_{E} A$. By decomposing these two matrices as

$$
X=U S U^{\top}, \quad Y=V H V^{\top}
$$

where $S$ and $H$ are real diagonal matrices. One can define

$$
\bar{B}=U|S|^{1 / 2}, \quad \bar{C}=|H|^{1 / 2} V^{\top} .
$$

Now, by writing Lyapunov equations with the newly defined vectors $\bar{B}$ and $\bar{C}$ as in (2.13), the solutions of these equations are the new controllability and observability grammians.

$$
\begin{aligned}
& A \hat{P}+\hat{P} A^{\top}=-\overline{B B}^{\top}, \\
& A^{\top} \hat{Q}+\hat{Q} A=-\bar{C}^{\top} \bar{C} .
\end{aligned}
$$

$\hat{P}$ and $\hat{Q}$ can be considered for model reduction. This method again guarantees the stability and preserves the performance for two sided weights, but no physical insight support the method.

In [5] another new method is introduced in order to enhance the model reduction performance. Their method is obtained by combining Enns and Lin methods to incorporate the advantage of both techniques. The grammians of their method are defined as

$$
\begin{aligned}
& P_{L}=P_{E}-\alpha_{c}^{2} P_{12} P_{22}^{-1} P_{12}^{\top}, \\
& Q_{L}=Q_{E}-\alpha_{o}^{2} Q_{12}^{\top} Q_{22}^{-1} Q_{12}
\end{aligned}
$$

These grammians are used for model reduction. If $\alpha_{c}=\alpha_{o}=0$, these grammians will be similar to Enns method and if $\alpha_{c}=\alpha_{o}=1$ the grammians will be like what Lin introduced.

In $[5,8]$ were suggested weights $W_{i}=G(I+G K)^{-1}$ and $W_{o}=(I+G K)^{-1}$ as performance preserving weights; these weights are calculated by forming the $\left\|K-K_{r}\right\|$ and neglecting terms of second order of $K-K_{r}$.

An alternative approach to model reduction method is Singular Perturbation Approximation (SPA) method; see
[5]. In SPA, after transforming the system into the form of $(2-5)$, the reduced order model can be calculated as

$$
\begin{aligned}
& A_{r}=A_{11}-A_{12} A_{22}^{-1} A_{21}, B_{r}=B_{1}-A_{12} A_{22}^{-1} B_{2}, \\
& C_{r}=C_{1}-C_{2} A_{22}^{-1} A_{21}, \quad D_{r}=D-C_{2} A_{22}^{-1} B_{2} .
\end{aligned}
$$

In SPA method, the DC gain of stable system is preserved. The conjunction of SPA and WBT leads to a new method which is named WBTSPA. This technique makes the approximation better in low frequency range and also preserves the stability.

All of the above mentioned methods are based on time-domain approaches, while in the new method which will be presented; the model reduction is performed in frequency-domain. The method is in the same line of [4]. In our method instead of performing the model reduction in the whole frequency range, only certain range of frequency which is of more importance will be considered. This frequency range can be selected to improve the reduction result. One advantage of the frequency domain approach for model reduction is that the weights can be two sided and even unstable and also for an unstable controller, the model reduction can be performed directly on the whole controller without decomposing it to stable and unstable parts at the expenses of lower accuracy.

\section{CLOSED LOOP CONTROLLABILITY AND OBSERVABILITY GRAMMIANS OF CONTROLLER}

In this section, new frequency domain characteristics based on energy distribution from the input to state and state to output view point are defined. These energy based characteristics which are called closed loop grammians of the controller will be used in this section for controller reduction.

Consider the closed loop system of Fig. 2. In this figure, the transfer function of the plant, $G(s)$ has a state space realization as equation $(2.1) . K(s)$ is a high order stable controllable and observable controller with state space realization as

$$
\begin{aligned}
\dot{x}_{k} & =A_{k} x_{k}+B_{k} u_{k}, \\
y_{k} & =C_{k} x_{k}
\end{aligned}
$$

where $x_{k} \in R^{n_{c}}$ is a state vector, $u_{k} \in R^{q}$ represents the input vector of the controller, and $y_{k} \in R^{q}$ is the output of the controller with matrices $A_{k}, B_{k}$, and $C_{k}$ in the appropriate dimensions and $r \in R^{q}$ is the reference input of the closed loop system. The order of the plant is $n$ and the order of controller is $n_{c}$.

The distribution of energy for the closed loop system between any two points on Fig. 2 in frequency domain can be obtained by defining proper transfer functions between these two points. To define energy distribution between reference input $r$ and controller state $x_{k}$, consider the following equations

$$
\begin{aligned}
& x_{k}=\left(s I-A_{k}\right)^{-1} B_{k} u_{k}, \\
& u_{k}=r-y=r-G C_{k} x_{k} .
\end{aligned}
$$


The substitution of these equations results

$$
\begin{aligned}
& x_{k}=\left(s I-A_{k}+B_{k} G C_{k}\right)^{-1} B_{k} r= \\
& \quad\left(s I-A_{k}\right)^{-1} B_{k}(I+G K)^{-1} r .
\end{aligned}
$$

Now, the energy distribution of the controller state from reference input can be calculated by performing the time integral. Its corresponding frequency integral (using Parsseval theorem) is given by

$$
E_{i}=\int_{0}^{\infty} x_{k}(t) x_{k}^{*}(t) \mathrm{d} t=\frac{1}{2 \pi} \int_{-\infty}^{\infty} x_{k}(\omega) x_{k}^{*}(\omega) \mathrm{d} \omega
$$

where

$$
x_{k}(\omega)=\left(j \omega I-A_{k}\right)^{-1} B_{k}(I+G K)^{-1} r .
$$

which corresponds to the state covariance matrix of controller $K(s)$.

Assuming that the reference input is white noise, the new closed loop controllability grammian of the controller $P_{k c}$ is defined as

$$
P_{k c}=\frac{1}{2 \pi} \int_{-\infty}^{\infty} \frac{x_{k}(\omega) x_{k}^{*}(\omega)}{r(\omega) r^{*}(\omega)} \mathrm{d} \omega
$$

This grammian is called frequency-domain closed loop controllability grammian of controller, and will be used for controller order reduction.

A similar interpretation for the controller state to the output of the closed loop system can be obtained. Consider the output of the closed loop system.

$$
y=G y_{k}=G C_{k} x_{k} .
$$

Then the energy distribution of output with respect to controller state can be written in time and frequency domains as

$$
E_{o}=\int_{0}^{\infty} y_{k}(t) y_{k}^{*}(t) \mathrm{d} t=\frac{1}{2 \pi} \int_{-\infty}^{\infty} y_{k}(\omega) y_{k}^{*}(\omega) \mathrm{d} \omega
$$

where

$$
x_{k}(\omega)=\left(j \omega I-A_{k}\right)^{-1} C_{k}(I+G K)^{-1} r .
$$

Again, by considering the input as white noise, the closed loop observability grammian of controller $Q_{k c}$ is defined as

$$
Q_{k c}=\frac{1}{2 \pi} \int_{-\infty}^{\infty} \frac{y_{k}(\omega) y_{k}^{*}(\omega)}{r(\omega) r^{*}(\omega)} \mathrm{d} \omega
$$

This grammian is called frequency-domain closed loop observability grammian of controller, and together with $P_{k c}$ will be used for controller order reduction.

Because most of the systems work in a certain frequency domain it is desirable to be able to tight the frequency domain and this can be easily done by limiting the frequency range of reference input signal. In this case, consider the input signal $r(j \omega)$ which its energy density spectrum is mainly confined in frequency range $\left[\omega_{0}, \omega_{1}\right]$ and zero elsewhere; that is

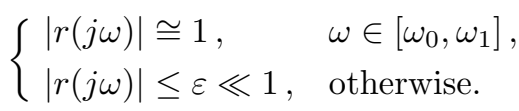

So the new controller controllability and observability grammians in equations (3.6) and (3.9) can also be defined in frequency range

$$
\begin{aligned}
P_{k c} & =\frac{1}{\pi} \int_{\omega_{0}}^{\omega_{1}} \frac{x_{k}(\omega) x_{k}^{*}(\omega)}{r(\omega) r^{*}(\omega)} \mathrm{d} \omega . \\
Q_{k c} & =\frac{1}{\pi} \int_{\omega_{0}}^{\omega_{1}} \frac{x_{k}(\omega) x_{k}^{*}(\omega)}{r(\omega) r^{*}(\omega)} \mathrm{d} \omega .
\end{aligned}
$$

These frequency weighted grammians provide flexibility for designer to select proper frequency range for controller order reduction so they can be used instead of the infinite frequency grammian $P_{k c}$ and $Q_{k c}$ in the procedure of order reduction.

The following lemma shows that by using a similarity transformation on the newly defined grammians, the eigenvalues of these grammians will be invariant so they can be used for controller reduction.

Lemma 3.1. For a linear time-invariant controller $\left(A_{k}, B_{k}, C_{k}\right)$ in a closed loop system as in Fig. 2, under a coordinate transformation such as $x_{k}(t)=T \tilde{x}_{k}(t)$ the following properties hold

i) The transformed closed loop controllability and observability grammians of the controller $\widetilde{P}_{k c}, \widetilde{Q}_{k c}$, satisfy the following relations

$$
\widetilde{P}_{k c}=T^{-1} P_{k c} T^{-1 *}, \widetilde{Q}_{k c}=T^{*} Q_{k c} T .
$$

ii) The singular values of the product $P_{k c}$ and $Q_{k c}$ that is, $\sigma_{i}=\sqrt{\lambda_{i}\left(P_{k c} Q_{k c}\right)}$ are invariant.

iii) There exists a specific transformation $\bar{T}$ such that the closed loop controllability and observability grammians of controller can be diagonolized and equal, that is, $\widetilde{P}_{k c}=\widetilde{Q}_{k c}=\Sigma_{f}$, where $\Sigma_{f}=\operatorname{diag}\left\{\sigma_{1}, \sigma_{2}, \ldots, \sigma_{n_{c}}\right\}$, $\sigma_{i} \geq \sigma_{i+1}, i=1,2, \ldots, n_{c}-1$.

Proof. i) Using the transformation $x_{k}=T \tilde{x}_{k}$ or $\tilde{x}_{k}=T^{-1} x_{k}$, the transformed controller matrices will be

$$
\widetilde{A}_{k}=T^{-1} A_{k} T, \widetilde{B}_{k}=T^{-1} B_{k}, \widetilde{C}_{k}=C_{k} T .
$$

Using these matrices for $\widetilde{P}_{k c}$ we have

$$
\widetilde{P}_{k c}=\frac{1}{2 \pi} \int_{\delta \omega} \widetilde{\xi}(\omega) \widetilde{\xi}(\omega)^{*} \mathrm{~d} \omega
$$

where $\delta \omega$ is the frequency range $\left[\omega_{0}, \omega_{1}\right]$, and where

$$
\begin{array}{r}
\widetilde{\xi}(\omega)=\left(j \omega I-\widetilde{A}_{k}+\widetilde{B}_{k} G \widetilde{C}_{k}\right)^{-1} \widetilde{B}_{k}= \\
=T^{-1}\left(j \omega I-A_{k}+B_{k} G C_{k}\right)^{-1} B_{k}=T^{-1} x_{k}(\omega)
\end{array}
$$




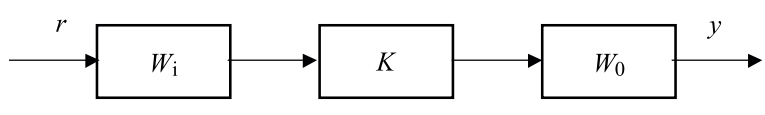

Fig. 3. Rearranged closed loop system

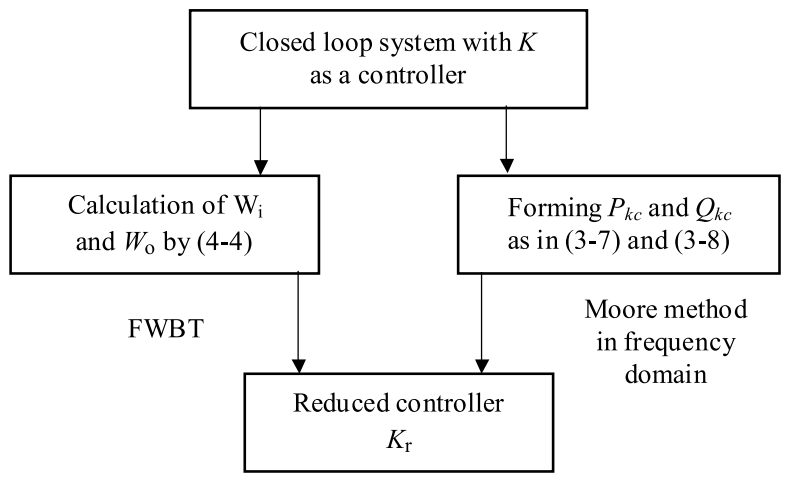

Fig. 4. Commutative diagram for the new method and Enns technique

hence

$$
\widetilde{P}_{k c}=\frac{1}{2 \pi} T^{-1} \int_{\delta \omega} x_{k}(\omega) x_{k}^{*} \mathrm{~d} \omega T^{-1 *}=T^{-1} P_{k c} T^{-1 *} .
$$

The second part for observability grammian can be proved similarly.

ii) $\widetilde{P}_{k c} \widetilde{Q}_{k c}=T^{-1} P_{k c} Q_{k c} T$, and the result follows.

iii) The proof is as the Moores, see [12].

Based on lemma 3.1, the singular values of the closed loop controllability and observability grammians of the controller are invariant under similarity transformation therefore the following results will be obtained

1) There is a coordinate transformation which can balance grammians. The procedure for calculating the coordinate transformation is presented in [12].

2) The magnitude of singular values represents the relative importance of the controller states from the closed loop system point of view.

Based on the relative magnitude of singular value $\Sigma_{f}$, the balanced controller and its grammians can be partitioned as

$$
\begin{gathered}
A_{k}=\left[\begin{array}{cc}
A_{k 11} & A_{k 12} \\
A_{k 21} & A_{k 22}
\end{array}\right], B_{k}=\left[\begin{array}{l}
B_{k 1} \\
B_{k 2}
\end{array}\right], C_{k}=\left[\begin{array}{ll}
C_{k 1} & C_{k 2}
\end{array}\right] \\
\Sigma_{f}=\left[\begin{array}{cc}
\Sigma_{f 1} & 0 \\
0 & \Sigma_{f 2}
\end{array}\right], X_{k}(t)=\left[\begin{array}{l}
x_{k 1}(t) \\
x_{k 2}(t)
\end{array}\right]
\end{gathered}
$$

where $A_{k 11}$ and $\Sigma_{f 1}$ are $r \times r\left(r<n_{c}\right)$ matrices. The system $\left(A_{k 11}, B_{k 1}, C_{k 1}\right)$ is the reduced- order controller.

The following steps described an algorithm for the proposed controller reduction method

1) Calculate closed loop controllability and observability grammians $P_{k c}$ and $Q_{k c}$ in the desired frequency range, from equations (3.11), (3.12).
2) Find a similarity transformation $T$ that makes the closed loop controller grammians balanced, that is, $P_{k c}=Q_{k c}=\Sigma_{f}$.

3) Partition the transformed controller as equation (3.13) based on the magnitude of grammian singular values. The subsystem $\left(A_{k 11}, B_{k 1}, C_{k 1}\right)$ is the reduced controller.

\section{PROPOSED APPROACH}

\subsection{General Properties}

Different interesting properties for the new method can be stated which are discussed in this section. The first property is the relation between the newly defined controller grammians in the closed loop system and the grammians of the closed loop transformation of Fig. 2. For the closed loop system, one can write a realization as

$$
\widetilde{A}=\left[\begin{array}{cc}
A_{k} & -B_{k} C_{G} \\
B_{G} C_{k} & A_{G}
\end{array}\right], \widetilde{B}=\left[\begin{array}{c}
B_{k} \\
0
\end{array}\right], \widetilde{C}=\left[\begin{array}{ll}
C_{k} & 0
\end{array}\right]
$$

and the corresponding Lyapunov equations for this system can be written as

$$
\begin{aligned}
\widetilde{A} P_{c}+P_{c} \widetilde{A}^{\top} & =-\widetilde{B} \widetilde{B}^{\top}, \\
\widetilde{A}^{\top} Q_{c}+Q_{c} \widetilde{A} & =-\widetilde{C}^{\top} \widetilde{C} .
\end{aligned}
$$

The solution of the first Lyapunov equation leads to

$$
P_{c}=\left[\begin{array}{cc}
P_{k c} & \int_{\omega_{0}}^{\omega_{1}} F_{1} B_{k} B_{k}^{\top} F_{1}^{\top} F_{2}^{\top} \mathrm{d} \omega \\
\int_{\omega_{0}}^{\omega_{1}} F_{2} F_{1} B_{k} B_{k}^{\top} F_{1}^{\top} \mathrm{d} \omega & P_{G c}
\end{array}\right]
$$

where $P_{G c}$ is the closed loop controllability grammian of the plant which can be defined as in (3.11)except that the plant transfer function should be considered instead of controller transfer function,

$P_{G c}=\int_{\omega_{0}}^{\omega_{1}} F_{2} F_{1} B_{k} B_{k}^{\top} F_{1}^{\top} F_{2}^{\top} \mathrm{d} \omega . F_{1}$ and $F_{2}$ are $F_{1}=$ $\left(j \omega I_{1}-A_{k}+B_{k} G C_{k}\right)^{-1}, F_{2}=\left(j \omega I_{2}-A_{G}\right)^{-1} B_{G} C_{k}$. An interesting observation is that the block (1-1) of $P_{c}$ is exactly the newly defined closed loop controllability grammian for controller. So for controller reduction of a closed loop system, one can easily use equation (3.10) instead of $n+n_{c}$ matrix of $P_{c}$ in frequency-weighted method. A similar conclusion can be drawn for the solution of the second Lyapunov equation in (4.2).

The second property is the relation between the new proposed method and frequency-weighted reduction of Enns. The closed loop system of Fig. 2 can be rearranged in the form of Fig. 3 where $W_{i}$ and $W_{o}$ can be obtained to be

$$
W_{i}=(I+G K)^{-1}, \quad W_{o}=G .
$$

By considering the $K W_{i}$ and $W_{o} K$ transfer functions, if one can calculate the controllability and observability grammians of frequency-weighted method of Enns as 
in (2.6); then the results will be exactly like (3.11) and (3.12). So the new method converges to Enns method but with new two sided weights $W_{i}$ and $W_{o}$ as defined in (4.4). This equivalence between newly defined method and Enns technique can be shown by a commutative diagram as in Fig. 4.

This diagram shows that the new method is exactly like Enns but with new weights and in frequency domain when the frequency range goes to infinite. The following theorem describes the result of the previous property.

THEOREM 4.1. The closed loop controller grammians of the new frequency domain controller reduction method (3.11) and (3.12) when the frequency range goes to infinite are equivalent to the grammians of Enns method with weights as defined in (4.4).

Proof. A sketch of the proof is described above.

In comparing the computational effort for the Enns FWBT method and the new method, one can see that in the new method we have to solve $n+n_{c}$ Lyapunov equations while in the Enns method, we have to solve $n+2 n_{c}$ Lyapunov equations. These extra equations corresponds to the plant's states, therefore in this case, unstable plants will results an improper Lyapunov equations.

One benefit of the new method is that most systems work in a desired frequency domain. Therefore, the equations (3.11) and (3.12) can be calculated only in certain frequency range and this can be done by selecting the desired upper and lower bound of integral. The flexibility in choosing the frequency band can help to have more accurate results. Another advantage of this method is that when the weights are not stable or the controller is not stable we still can use the finite frequency range method but with less accuracy.

\section{Stability}

The stability property of the reduced order controller is an important issue which will be discussed in this subsection. Enns showed that frequency weighted balanced truncation result in a stable reduced order model when the weights are one sided, ie $W_{i}=I$ and $W_{o} \neq I$ or $W_{i} \neq I$ and $W_{o}=I$. As we showed in this section, the new method is equivalent to Enns, therefore the stability of newly proposed method is already proved for one sided weights. Here, we will show that frequency- domain closed loop grammians $P_{k c}$ and $Q_{k c}$ satisfy Lyapunovtype equations, so that stability property of controller reduction method is verified. Because of equivalence with Enns, this result can be extended to show that frequency weighted reduction method of Enns yields a stable reduced model even with two sided weights.

Consider a stable band pass filter $W(\omega)$ as

$$
\begin{cases}W(\omega) \cong 1, & \omega_{0}<\operatorname{abs}(\omega)<\omega_{1}, \\ W(\omega)<\varepsilon \ll 1, & \text { elswhere. }\end{cases}
$$

Then, the finite frequency domain closed loop controllability grammian $P_{k c}$ in equation $(3.11)$ can be written approximately as

$$
\begin{aligned}
\hat{P}_{k c}=\frac{1}{2 \pi} \int_{-\infty}^{\infty}\left\{W\left(j \omega I-A_{k}\right)^{-1}\right. & \left\{W B_{k} W_{i} W_{i}^{*} B_{k}^{*}\left(j \omega I-A_{k}\right)^{-1 *} W^{*}\right\} \mathrm{d} \omega \\
& \times
\end{aligned}
$$

where $W_{i}$ is any desired weight or specific weight $W_{i}=$ $(I+G K)^{-1}$.

Here, the range of integration has changed because of application of bandpass filter $W(\omega)$. Using Parsseval theorem, (4.6) can be rewritten in time domain as:

$$
P_{k c}(t)=\int_{0}^{\infty} W \otimes e^{A_{*} t} B_{k} \otimes W_{i} W^{*} B_{k}^{*} e^{A_{k}^{*} t} \otimes W^{*} \mathrm{~d} t
$$

where the symbol $\otimes$ denotes time convolution. By forming the Lyapunov equation for the controllability grammian of the controller we have

$$
\begin{aligned}
& A_{k} P_{k c}+P_{k c} A_{k}^{\top}= \\
& =A_{k} \int_{0}^{\infty} e^{A_{k} t} B_{k} \otimes W \otimes W_{i} W_{i}^{*} \otimes W^{*} \otimes B_{k}^{*} e^{A_{k}^{*} t} \mathrm{~d} t \\
& +\int_{0}^{\infty} e^{A_{k} t} B_{k} \otimes W \otimes W_{i} W_{i}^{*} \otimes W^{*} \otimes B_{k}^{*} e^{A_{k}^{*} t} \mathrm{~d} t A_{k}^{\top} \\
& =\int_{0}^{\infty} \frac{\mathrm{d}}{\mathrm{d} t}\left[e^{A_{k} t} B_{k} \otimes W \otimes W_{i} W_{i}^{*} \otimes W^{*} \otimes B_{k}^{*} e^{A_{k}^{*} t}\right] \mathrm{d} t \\
& \quad=\int_{0}^{\infty} \frac{\mathrm{d}}{\mathrm{d} t}\left[z z^{*}\right] \mathrm{d} t=z(\infty) z^{*}(\infty)-z(0) z^{*}(0)
\end{aligned}
$$

where $z=W \otimes e^{A_{k} t} B_{k} \otimes W_{i}$.

If the original controller is stable, the states of the controller converge to zero as time goes to infinity, so $z(\infty) \rightarrow 0$ and we have

$$
A_{k} P_{k c}+P_{k c} A_{k}^{\top} \leq 0 \Longrightarrow A_{k} P_{k c}+P_{k c} A_{k}^{\top}=-L L^{*} \text {. }
$$

So the controllability Lyapunov equation for controller is negative semi-definite. By a similar procedure, it can be shown that the observability Lyapunov equation for controller is also negative semi-definite, that is,

$$
A_{k}^{*} Q_{k c}+Q_{k c} A_{k}=-N N^{*} .
$$

Since $P_{k c}$ and $Q_{k c}$ satisfy time Lyapunov equations (however with different $B_{k}$ and $C_{k}$ ), the stability of the reduced order controller is automatically guaranteed, see [13]. The following theorem mimics the stability result of the reduced order controller.

TheOrem 4.2. For a stable, controllable and observable controller with stable input and output weights, the reduced order controller with grammians $P_{k c}$ and $Q_{k c}$ is stable.

Pro of. The above described procedure proves the result.

In general, if the frequency bound is not finite, the frequency window $W(\omega)$ is not needed in stability proof and the stability of two sided weight model reduction for Enns technique can also be insured. 
Table 1. The comparison of error of different methods

\begin{tabular}{cc}
\hline Method & Error \\
New method & 0.2944 \\
Wang & 0.3973 \\
Anderson $(\alpha=0.5)$ & 0.4812 \\
SPA & Unstable \\
Lin & $\mathrm{N} / \mathrm{A}$ \\
\hline
\end{tabular}

Table 2. The comparison of new weights and Anderson weights with different methods

\begin{tabular}{ccc}
\hline Method & Weights 1 & Weights 2 \\
New Method \& Enns & 0.0982 & 0.1155 \\
Wang & 2.2532 & 31.2144 \\
Anderson $(\alpha=0.5)$ & 2.3937 & 2.1684 \\
WBTSPA & Unstable & Unstable \\
Lin & NA & NA \\
\hline
\end{tabular}

\section{SIMULATION RESULTS}

In this section we present a numerical example to show the effectiveness of new method. The example is taken from [14] which the control design for the plant would lead to an unstable controller. The example highlight different aspects of the proposed method; in the first part is compared with other controller reduction methods to show a better error performance; the second part emphasizes the choice of input and output weight and at last the capability of method to deal directly with unstable controller reduction, instead of partitioning it to stable and unstable parts is shown. Consider the plant $G(s)$ in Fig. 2 with a minimal realization as

$$
\begin{gathered}
A_{G}=\left[\begin{array}{ccc}
-1 & 0 & 4 \\
0 & -2 & 0 \\
0 & 0 & -3
\end{array}\right], \quad B_{G}=\left[\begin{array}{l}
1 \\
1 \\
1
\end{array}\right] \\
C_{G}=\left[\begin{array}{lll}
1 & 1 & 1
\end{array}\right], \quad D_{G}=0
\end{gathered}
$$

Using the robust control technique, a controller with the following transfer function has been designed in [14].

$$
K=\frac{148.79(s+1)(s+3)}{(s+31.74)(s+3.85)(s-9.19)} .
$$

PART I. In order to be able to compare our method with other controller reduction methods, it is necessary to partition the controller to two parts, one part which is stable and the other which is unstable

$$
K_{1}=\frac{114.15(s+3.61)}{(s+31.74)(s+3.85)}, \quad K_{2}=\frac{34.64}{s-9.19} .
$$

The new closed loop grammian of the controller in the frequency range of 2 to $100 \mathrm{~Hz}$ are

$$
P_{k}=\left[\begin{array}{ll}
1.2367 & 2.4232 \\
2.4232 & 8.0501
\end{array}\right], \quad Q_{k}=\left[\begin{array}{ll}
0.1725 & 0.0273 \\
0.0273 & 0.0044
\end{array}\right]
$$

which has singular values $\left\{\sigma_{1}^{2}=0.3806, \sigma_{2}^{2}=0.0001\right\}$. Using the proposed algorithm in section 4 , the reduced order controller is given as

$$
K_{1 r}=\frac{105.3}{s+28.8}
$$

The controller reduction with other methods has been performed and the error for different method is summarized in Tab. 1. The error is obtained from $e=$ $\left\|H-H_{r}\right\|_{\infty}$, where $H$ the transfer function of the closed loop system in Fig. 2 with original controller and $H_{r}$ is the closed loop transfer function with the reduced order controller.

In Tab. 1, the weights for Wang and Anderson methods have been selected from Enns one sided weights, that is, $W_{i}=G(I+G K)^{-1}, W_{o}=I$. It is obvious that the new method has better result than the other methods.

PART II. In this part the choice of $W_{i}$ and $W_{o}$ on the controller reduction problem will be studied. Two sided weights are considered; the first choice is the newly proposed weights $W_{i}=G$ and $W_{o}=(I+G K)^{-1}$ which were obtained in the procedure of controller reduction and the second choice are the Anderson performance preserving weights; $W_{i}=(I+G K)^{-1}$ and $W_{o}=(I+G K)^{-1} G$.

The errors for these two choices of weights are shown in Tab. 2. This table shows better performance of newly defined weights in controller reduction techniques.

PART III. The last part is intended to show that the newly controller reduction with finite frequency domain consideration can be implemented for unstable controller directly instead of partitioning it. The reduced order controller can be obtained directly from finite frequency grammians (3.11) and (3.12) as

$$
K_{r}=\frac{124.1 s+89.94}{s^{2}+27.93 s-341.1}
$$

with an error of $\left\|H-H_{r}\right\|_{\infty}=7.4608$; as it is clear the error is larger than the case of partitioning the controller. So, although direct implementation of method is applicable on the unstable controller, but it is not recommended.

\section{CONCLUSIONS}

In this paper a new frequency based controller reduction method was proposed. The method was based on new frequency-domain controllability and observability grammians. The relation of this method and Enns was introduced and the stability of the method was discussed. The simulation results show the effectiveness of this method. 


\section{REFERENCES}

[1] LIN, C. A.-CHIN, T. Y.: Model Reduction via Frequency Weighted Balanced Realization, Control Theory and Advanced Technol. 8 (1992), 341-351.

[2] SCHELfHOUT, G.-MOOR, B. D.: A Note on Closed-Loop Balanced Truncation, IEEE Trans. Auto. Control 41 (1996), $1498-1500$.

[3] WANG, G.-SREerAM, V.-LIU, W. Q.: A New Frequency-Weighted Truncation Method and an Error Bound, IEEE Trans. Auto. Control 44 No. 9 (1999), 1734-1737.

[4] KARIMAGHAEE, P.-ZILOUCHIAN, A.-NIKE-RAVESH, S.-ZADEGAN, A. H.: Principle of Frequency-Domain Balanced Structure in Linear Systems and Model Reduction, Computers and Electrical Eng. 29 (2002), 463-477.

[5] VARGA, A.-ANDERSON, B. D. O.: Accuracy-Enhancing Methods for Balancing-Related Frequency-Weighted Model and Controller Reduction, Automatica 39 (2003), 919-927.

[6] GUGERCIN, S. : Controller Reduction for Large-Scale Systems by Krylov Projection Methods, SIAM Conference on Computational Science and Engineering, Orlando, FL, USA, 2005.

[7] HOULIS, P.-SREERAM, V.: A Parametrized Controller Reduction Technique, Proceedings of the 45th IEEE Conference on Decision \& Control, Manchester Grand Hyatt Hotel San Diego, CA, USA, 2006, pp. 13-15.

[8] ANDERSON, B. D. O.-LIU, Y.: Controller Reduction: Concepts and Approaches, IEEE Trans. Automat. Contr. 34 (1989), 802-812.

[9] ENNS, D. F. : Model Reduction with Balanced Realization, An Error Bound and Frequency Weighted Generalization, in 23rd Conf. Decision Control, Las Vegas, NV, 1984, pp. 1237-1321.

[10] YOUSEFI, A.-LOHMANN, B.: Stabilizing Frequency Weighted Controller Reduction Approach, ISA Transactions 46 (2007), 223-231.

[11] GHAFOOR, A.-SREERAM, V.: Frequency Weighted Balanced Model Reduction: A Survey, ICARCV Singapore, 2006.

[12] MOORE, B. C. : Principal Component Analysis in Linear Systems: Controllability, Observability and Model Reduction, IEEE Trans. Automat. Contr. 26 (1981), 17-32.
[13] PERNABO, L.-SILVERMAN, L. M.: Model Reduction via Balanced State Space Representations, IEEE Trans. Auto. Control 27 (1982), 382-387.

[14] ZHOU, K.-DOYLE, J. C.-GLOVER, K. : Robust and Optimal Control, Prentice-Hall, New York.

Received 15 May 2009

Roozbeh Sadeghian was born in Isfahan, Iran,on May 1979 . He received the BS degree in Electrical Engineering in 2001 from Isfahan industrial University, Isfahan, and his MS degrees in electrical engineering in 2005 from Shiraz university, Shiraz, Iran. He is currently with International System Engineering and Automation Company (IRISA) at Isfahan. His research interests include controller order reduction and industrial automation.

Paknosh Karimaghaee was born in Shiraz, Iran,on September 23, 1967. He received the BS degree in Electrical Engineering in 1992 from Shiraz University, Shiraz, Iran, and his $\mathrm{MS}$ and $\mathrm{PhD}$ degrees in electrical engineering from Amirkabir University of Technology (AUT) in 1995 and 2001 respectively. He is currently Assistant Professor in electrical engineering at Shiraz university. His research interests include model and controller reduction,nonlinear systems analysis, control of discrete event systems, and hybrid systems

Alireza Khayatian received the BSc Degree in Electrical Engineering from Isfahan Technical University (Iran) in 1985, and the MSc degree also in Electrical Engineering from Tarbiat Modarres University (Iran) in 1988. He was a Research Assistant in the Electromechanical Systems Laboratory at the Georgia Institute of Technology where, he received his $\mathrm{PhD}$ degree in Control Engineering in 1993. Since 1993, he has been a faculty member with the Department of Electrical Engineering, Shiraz University, Shiraz, Iran and his research interests include Nonlinear Control, Estimation and Robotics.

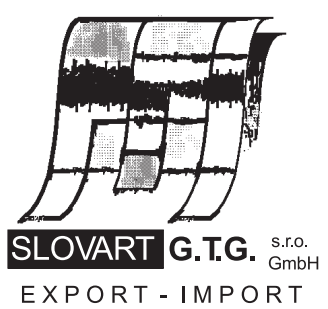

EXPORT - IMPORT
EXPORT - IMPORT

of periodicals and of non-periodically printed matters, books and CD-ROMs

Krupinská 4 PO BOX 152, 85299 Bratislava 5, Slovakia tel: ++421263839 472-3, fax: ++421263839485 info@slovart-gtg.sk; http://www.slovart-gtg.sk

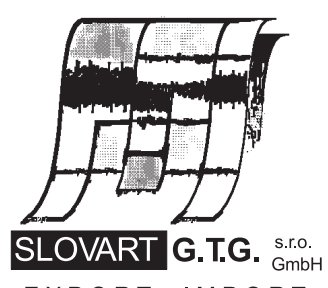

EXPORT - IMPORT 